\title{
TÉCNICAS DE ATERRISSAGEM AFETAM O DESEMPENHO E ESTRESSE MECÂNICO DURANTE DROP JUMP
}

\author{
LANDING TECHNIQUES AFFECT PERFORMANCE AND MECHANICAL STRESS DURING DROP JUMP
}

TÉCNICAS DEATERRIZAJEAFECTAN EL RENDIMIENTO Y LATENSIÓN MECÁNICA DURANTE

Artigo Original

Original ARtICle

Artículo Original

EL SALTO DROP JUMP

Paulo Henrique Marchetti $1^{1,6}$

(Profissional de Educação Física)

Thaís Tomaz dos Santos ${ }^{1}$

(Profissional de Educação Física)

Enrico Gori Soares ${ }^{1,3}$

(Profissional de Educação Física)

Erica Paes Serpa ${ }^{1,4}$

(Profissional de Educação Física)

Josinaldo Jarbas da Silva ${ }^{1,5}$

(Profissional de Educação Física)

Roberto Aparecido Magalhães

(Profissional de Educação Física)

Marcelo Massatoshi Senaga Miyatake (Profissional de Educação Física)

Guanis de Barros Vilela Junior ${ }^{1}$ (Profissional de Educação Física)

Willy Andrade Gomes ${ }^{1,2}$

(Profissional de Educação Física)

1. Universidade Metodista de Piracicaba (UNIMEP), Faculdade de Ciências da Saúde (FACIS), Programa de Pós-Graduação Stricto Sensu em Ciências do Movimento Humano, Piracicaba, SP, Brasil. 2. Universidade Nove de Julho (UNINOVE), Programa de Graduação em Educação Física, São Paulo, SP, Brasil.

3. Centro Regional Universitário de Espírito Santo do Pinhal (UNIPINHAL), Programa de Graduação em Educação Física, Espírito Santo do Pinhal, SP, Brasil. 4. Faculdade de Ciências Sociais e Agrárias de Itapeva (FAIT), Programa de Graduação em Educação Física, Itapeva, SP, Brasil.

5. Faculdades Integradas do Vale do Ribeira (FVR), Programa de Graduação em Educação Física, Registro, SP, Brasil.

6. Universidade de São Paulo, Faculdade de Medicina, Instituto de Ortopedia e Traumatologia, São Paulo, Brasil.

\section{Correspondência:}

Universidade Metodista de Piracicaba, Faculdade de Ciências da Saúde, Programa de PósGraduação em Ciências do Movimento Humano, Grupo de Pesquisa em Performance Humana. Rodovia do Açúcar Km 156, Bloco 7 , Sala 42, Taquaral, Piracicaba, SP, Brasil. 13423-070. dr.pmarchetti@gmail.com

\section{RESUMO}

Introdução: O dropjumpé utilizado para melhorar da potência dos membros inferiores. Mantendo-se a altura de queda constante, a variação da técnica de aterrissagem pode alterar o desempenho e as características mecânicas do salto. Objetivo: Avaliar os efeitos da alteração da técnica de aterrisagem no desempenho e na força de reação do componente vertical do solo após drop jump. Métodos: Vinte e cinco homens saudáveis e fisicamente ativos (idade: $26 \pm 8$ anos, massa corporal total: $75 \pm 10 \mathrm{~kg}$, altura: $175 \pm 10 \mathrm{~cm}$ ) realizaram quatro técnicas de aterrisagem após o drop jump: bounce drop jump (BDJ), drop jump com $90^{\circ}$ de flexão do joelho (DJ90), drop jump com $135^{\circ}$ de flexão do joelho (DJ135) e drop jump com $135^{\circ}$ de flexão do joelho e com apoio das mãos no solo (DJ135A). Foram realizadas três tentativas para cada técnica, os sujeitos realizaram o drop jump caindo de uma plataforma de força de $40 \mathrm{~cm}$ de altura. As medidas de altura de salto, tempo de contato e impacto foram determinadas através da força de reação do componente vertical do solo, medida pela plataforma de força, em frequência de aquisição de $2.000 \mathrm{~Hz}$. A ANOVA com medidas repetidas foi utilizada para comparar as diferentes técnicas. Resultados: A técnica BDJ apresentou menor altura de salto, menor tempo de contato e maior impacto que as demais condições $(P<0,001)$. A técnica DJ90 apresentou altura de salto similar às condições DJ135 e DJ135A e tempo de contato menor que as condições DJ135 e DJ135A, além de menor impacto que as demais condições $(P<0,001)$. Conclusão: As maiores alturas do salto vertical foram observadas para DJ90, DJ135 e DJ135A. O menor tempo de contato foi observado na técnica BDJ, seguido de DJ90. Quanto ao impacto, a técnica BDJ apresentou os maiores valores.

Descritores: desempenho atlético; força muscular; fenômenos biomecânicos.

\section{ABSTRACT}

Introduction: The drop jump is used to improve the power of the lower limbs. Keeping the drop height constant, the variation of the landing technique can modify the performance and the mechanical characteristics of the jump. Objective: To evaluate the effects of different landing techniques on the performance and the reaction force of the vertical component of the ground after drop jump. Methods: Twenty-five healthy and physically active men (age: $26 \pm 8$ years, total body mass: $75 \pm 10 \mathrm{~kg}$, height: $175 \pm 10 \mathrm{~cm}$ ) performed four landing techniques after the drop jump: bounce drop jump (BDJ), drop jump with $90^{\circ}$ of kneeflexion (DJ90), drop jump with $135^{\circ}$ of kneeflexion (DJ135), and drop jump with $135^{\circ}$ of knee flexion with hands resting on the ground (DJ135A). Three attempts were performed for each technique; the subjects performed the drop jump falling from a $40 \mathrm{~cm}$ high force platform. The measures of jump height, contact time and impact were determined by the force of reaction of the vertical component of the ground, measured by the force platform, at a frequency of acquisition of 2,000 Hz. The repeated measures by ANOVA were used to compare the different techniques. Results: The BDJ technique presented lower jump height, shorter contact time and greater impact than the other techniques $(P<0.001)$. The DJ90 technique presented jump height similar to the DJ135 and DJ135A techniques and a short contact time $(P<0.001)$ and a lower impact than the other techniques $(P<0.001)$. Conclusion: The highest jumps were observed for DJ90, DJ135 and DJ135A. The lowest contact time was observed in the BDJ technique, followed by $D J 90$. Regarding the impact, the BDJ technique presented the highest values.

Keywords: athletic performance; muscle strength; biomechanical phenomena.

\section{RESUMEN}

Introducción: El drop jump se utiliza para mejorar la potencia de las extremidades inferiores. Manteniéndose la altura de caída constante, la variación de la técnica de aterrizaje puede cambiar el rendimiento y las características mecánicas del salto. Objetivo: Evaluar los efectos del cambio de técnica de aterrizaje en el rendimiento y en la fuerza de reacción del componente vertical del suelo después del drop jump. Métodos: Veinticinco hombres sanos y físicamente activos (edad: $26 \pm 8$ años, índice de masa corporal: $75 \pm 10 \mathrm{~kg}$, altura: $175 \pm 10 \mathrm{~cm}$ ) realizaron cuatro técnicas de aterrizaje después del drop jump: bounce drop jump (BDJ), drop jump flexionando las rodillas a 90 (DJ90), drop jump flexionando las rodillas a $135^{\circ}$ (DJ135), y drop jump flexionando las rodillas a $135^{\circ}$ con apoyo de las manos en el suelo (DJ135A). Se hicieron tres intentos para cada técnica; los sujetos realizaron el drop jump desde una plataforma de fuerza de $40 \mathrm{~cm}$. La medición de la altura del salto, el tiempo de contacto y el impacto se determinaron por la fuerza de reacción del componente vertical del suelo, medida por la plataforma de fuerza, en frecuencia de adquisición de 
$2.000 \mathrm{~Hz}$. Un ANOVA de medidas repetidas se utilizó para comparar las diferentes técnicas. Resultados: La técnica BDJ mostró menor altura del salto, menos tiempo de contacto y un mayor impacto que las otras condiciones $(P<0,001)$. La técnica DJ90 presentó un salto de altura similar a las condiciones de DJ135 y DJ135A y menos tiempo de contacto que las condiciones de DJ135 y condiciones DJ135A, e impacto más bajo que las otras condiciones $(P<0,001)$. Conclusión: Las mayores alturas de salto vertical se observaron en DJ90, DJ135 y DJ135A. El tiempo de contacto más bajo fue el de la técnica BDJ, seguida de DJ90. En cuanto al impacto, la técnica BDJ presentó los valores más altos.

Descriptores: rendimiento atlético; fuerza muscular; fenómenos biomecánicos.

\section{INTRODUÇÃO}

O exercício drop jump e suas variações são frequentemente incorporados às rotinas do treinamento de modalidades dependentes da produção de potência nos membros inferiores ${ }^{1-3}$. 0 drop jump é caracterizado pela queda de uma altura pré-determinada, seguida imediatamente de um salto máximo. A rápida transição entre ações excêntrica e concêntrica minimiza o tempo de contato com o solo e potencializa os mecanismos do ciclo alongamento-encurtamento (CAE), garantindo máximo desempenho durante a fase de propulsão (concêntrica) ${ }^{4}$. A literatura sugere que os mecanismos responsáveis pela melhora do desempenho na fase concêntrica são: (I) pré-ativação das unidades motoras, (II) maior ativação muscular decorrente do reflexo miotático, (III) acúmulo e utilização da energia elástica muscular e (iv) maior tempo para produção de força ${ }^{4-6}$.

Apesar dos mesmos mecanismos serem observados em outros exercícios de salto, o drop jump destaca-se entre os exercícios pliométricos por possibilitar a manipulação da altura de queda. A manipulação da altura de queda é considerada como a principal maneira de controlar a intensidade do exercício afetando diretamente o desempenho final $\left.\right|^{5,7,8}$. A fim de maximizar a intensidade do exercício, a literatura sugere que a altura de queda seja individualizada, sendo sempre utilizada aquela que garanta maior altura de salto $3,9,10$.

Por outro lado, entende-se que a utilização de uma grande intensidade gera maior estresse mecânico, e que pode, no treinamento, estar associado a uma grande frequncia de estímulos repetidos, ocasionando maior probabilidade de lesões no tecido biológico dos membros inferiores ${ }^{5}$. Desta forma, uma possível estratégia para manipular a magnitude de estresse mecânico é manipular a técnica de aterrissagem do salto, principalmente regulando a amplitude de deslocamento tanto do centro de massa quanto a de membros inferiores. Fischer et al. ${ }^{11}$, demonstraram que o pico de força no agachamento isométrico correlaciona-se com o pico de flexão do joelho durante a aterrissagem. Portanto, indivíduos mais fracos tendem a flexionar menos os joelhos durante o drop jump. Devita e Skelly ${ }^{12}$ e posteriormente Zhang et al. ${ }^{13}$, demonstraram que as aterrissagens mais suaves são realizadas com maiores ângulos de flexão de joelho. Entretanto, ambos os estudos atentaram-se somente a fase de aterrissagem sem verificar seu efeito no desempenho do salto. Adicionalmente, pouco se sabe sobre a relação entre o desempenho máximo e a técnica aterrissagem realizada. Sendo assim, o objetivo do presente estudo foi avaliar os efeitos no desempenho e na força de reação do solo vertical utilizando diferentes técnicas de aterrissagem após o drop jump.

\section{MÉTODOS}

\section{Amostra}

A amostra foi composta por 25 adultos saudáveis e fisicamente ativos (idade: $26 \pm 8$ anos, massa: $75 \pm 10$ kg, estatura: $175 \pm 10 \mathrm{~cm}$ ). Os sujeitos selecionados não apresentavam histórico de lesões osteomioarticulares e/ou cirurgia prévia nos membros superiores e inferiores e na coluna. 0 estudo foi aprovado pelo comitê de ética em pesquisa da universidade
(Protocolo\#11/2013), todos os participantes da pesquisa assinaram o Termo de Consentimento Livre e Esclarecido. O tamanho da amostra foi determinado a partir de um estudo piloto previamente realizado, a partir do teste de altura de salto e utilizando sujeitos com as mesmas características do presente estudo, baseando-se em uma significância de $5 \%$ e um poder de teste de $80 \%$.

\section{Procedimentos}

Após realizar um breve aquecimento composto de saltos submáximos, os sujeitos foram familiarizados com os procedimentos experimentais. O drop jump foi realizado a partir da queda de uma plataforma de $40 \mathrm{~cm}$ de altura, em que os sujeitos aterrissaram sobre uma plataforma de força (EMG System do Brasil, São José dos Campos, Brasil). Então, os sujeitos foram orientados a realizar quatro técnicas distintas de aterrissagem, seguidas de um salto máximo. Os dados da força de reação do solo vertical (FRSv) foram adquiridos a uma frequência de aquisição de $2000 \mathrm{~Hz}$, utilizando um software comercial (DATAQ Instruments Hardware Manager, DATAQ Instruments, Inc., OH, USA).

Assim, as quatro técnicas de aterrissagem foram testadas de forma aleatória: bounce drop jump (BDJ), técnica que enfatiza a ação no tornozelo (priorizando os flexores plantares) envolvendo mínima flexão do joelho e tempo de contato; drop jump com aterrissagem e flexão do joelho até $\sim 90^{\circ}$ (DJ90); drop jump com aterrissagem e flexão do joelho até $\sim 135^{\circ}$ (DJ135) e drop jump com aterrissagem e flexão do joelho até $\sim 135^{\circ} \mathrm{e}$ apoiando as mãos no chão (DJ135A). Foram realizados três saltos por técnica de aterrissagem, com um minuto de intervalo entre os saltos e três minutos entre as técnicas. Para minimizar o auxílio dos membros superiores, os sujeitos foram orientados a manter as mãos apoiadas sobre a crista ilíaca durante todo o tempo (exceto para a técnica DJ135A, durante a aterrissagem), e a realizar um esforço máximo em todos os saltos, além de tentar minimizar o tempo de contato com o solo em todas tentativas. Todos os sujeitos utilizaram seus próprios calçados.

\section{Análise dos dados}

A FRSv foi filtrada com um filtro passa-baixa Butterworth de quarta-ordem a $100 \mathrm{~Hz}$. As variáveis dependentes analisadas para todas as técnicas foram: (1) Altura do salto (em cm), a qual foi calculada a partir da velocidade de takeoff $\left(v_{\text {takeoff }}\right)$ do centro de massa pela fórmula:

$$
V_{\text {takeoff }}^{2} /(2 g)
$$

sendo g a aceleração da gravidade $\left(9,8 \mathrm{~m} / \mathrm{s}^{2}\right)$; (2) Tempo de contato que foi definido pelo tempo de apoio do sujeito na plataforma de força; (3) Pico de impacto (XPC) foi definido como o maior valor da curva força-tempo via FRSv, e normalizado pelo peso corporal de cada sujeito.

\section{Análise estatística}

A normalidade e homogeniedade das variâncias foram confirmadas pelos testes de Shapiro-Wilk e Levene, respectivamente. Para a comparação das variáveis dependentes para as diferentes técnicas de aterrissagem após o drop jump (BDJ, DJ90, DJ135 e DJ135A), uma 
ANOVA de medidas repetidas foi empregada. Um post-hoc de Bonferroni (com correção) foi utilizado para verificar as diferenças entre as técnicas. O tamanho do efeito (TE) foi calculado a partir da formula de Cohen e os resultados basearam-se nos seguintes critérios: <0.35 efeito trivial; 0.35-0.80 efeito pequeno; 0.80-1.50 efeito moderado; e $>1.5$ grande efeito, para sujeitos recreacionalmente treinados de acordo com Rhea ${ }^{14}$. A reprodutibilidade das tentativas foi calculada a partir do coeficiente de correlação intraclasse $(\mathrm{CCl})$ e foi baseado nos seguintes critérios: $<0.4$ pobre; $0.4-<0.75$ satisfatório; $\geq 0.75$ excelente ${ }^{15}$. Para todas as variáveis dependentes os CCI variaram entre 0.84 e 0.98. Uma significância de 5\% foi utilizada para todos os testes através do software SPSS versão 20.0.

\section{RESULTADOS}

A altura de salto na técnica BDJ foi significantemente menor em comparação com as demais técnicas (BDJ x DJ90: $P=0,001$; BDJ x DJ135: $P=0,001$; BDJ x DJ135A: $P=0,001)$. As técnicas DJ90, DJ135 e DJ135A não apresentaram diferenças significantes entre si (Figura 1A).

O tempo de contato na técnica BDJ foi significantemente menor em comparação com as demais (BDJ x DJ90: $P=0,001$; BDJ xDJ135: $P=0,001$, BDJ x DJ135A: $P=0,001$, DJ90 x DJ135: $P=0,001 ; D J 90 \times D J 135 A: P=0,034)$. Adicionalmente, a técnica DJ90 apresentou menor tempo de contato que as condições DJ135 e DJ135A, sendo que estas não diferiram significantemente entre si (Figura 1B).

O menor pico de impacto foi observado na técnica DJ90 diferindo significantemente das demais (BDJ XDJ90: $P=0,001$; BDJ $\times$ DJ135: $P=0,024$; DJ90 x DJ135: $P=0,001 ; D J 90 \times$ DJ135A: $P=0,001)$. A técnica DJ135 apresentou menor pico de impacto em relação à técnica BDJ. Não foram observadas diferenças significantes entre as outras técnicas (Figura 1C).

A comparação do tamanho do efeito e a variação percentual das variáveis dependentes são apresentadas na Tabela 1.

\section{DISCUSSÃO}

Os resultados do presente estudo demonstram diferenças evidentes no desempenho e na resposta da força de reação do solo vertical decorrentes da alteração da técnica de aterrissagem após o drop jump. Considerando a relação entre desempenho $x$ estresse mecânico, a técnica DJ90 apresentou ser a mais eficiente, pois aliou os maiores resultados em altura de salto e o menor pico de impacto em relação às demais técnicas. As técnicas DJ135 e DJ135A apresentaram alturas de salto similares à técnica DJ90, no entanto, apresentaram maior pico de impacto e maior tempo de contato do que o DJ90. Apesar da técnica BDJ apresentar menor tempo de contato, a mesma apresentou menor altura de salto e maior pico de impacto do que às demais técnicas.

Os melhores desempenhos para altura de salto vertical foram observados nas técnicas DJ90, DJ135 e DJ135A em comparação a BDJ. É provável que a técnica BDJ propicie menor tempo para produção de impulso, já que apresentou menor deslocamento vertical e menor tempo de contato. Além disso, o BDJ apresentou menor amplitude articular de membros inferiores do que as demais técnicas (DJ90, DJ135 e DJ135A), o que pode ter restringido a contribuição da musculatura envolvida em cada articulação durante a fase de propulsão do salto (concêntrica). De fato, McBride at al. ${ }^{16}$, demonstraram que o impulso produzido na fase concêntrica do salto com contra-movimento é o maior determinante do desempenho vertical em diferentes amplitudes de flexão do joeIho. Adicionalmente, Bobbert et al. ${ }^{17}$, demonstraram que o quadril e o joelho contribuem em aproximadamente 33 e 37\% respectivamente no trabalho total realizado durante o drop jump (40 cm de altura), com uso de técnica auto-selecionada, sendo o tornozelo responsável por $30 \%$ na contribuição total. Posteriormente, Lees et al. ${ }^{18}$, demonstraram que a contribuição do trabalho total das articulações do tornozelo e do joelho não é alterada entre saltos com contra-movimento submáximos e máximos. No entanto, a contribuição da extensão do quadril aumentou.
A

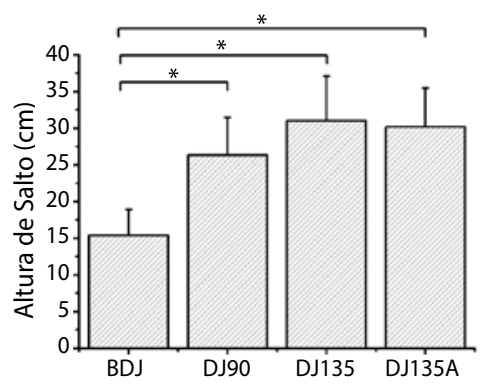

B

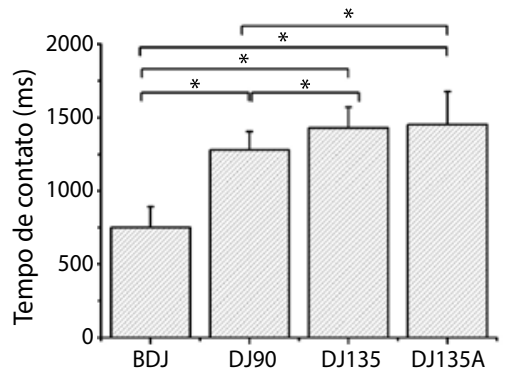

C

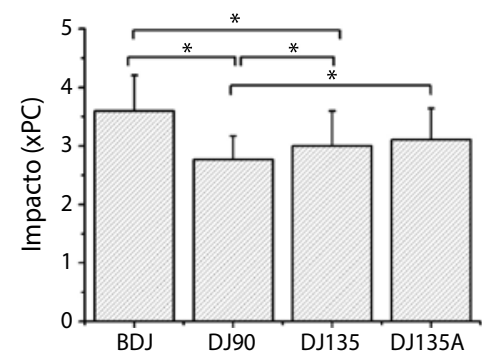

$B D J=$ bouncedrop jump; $\mathrm{DJ} 90=$ drop jump com aterrissagem de $\sim 90^{\circ}$ de flexão do joelho; $D J 135=$ drop jump com aterrissagem de $\sim 135^{\circ}$ de flexão do joelho e DJ135A=drop jump com aterrissagem de $\sim 135^{\circ}$ de flexão de joelho e apoiando as mãos no chäo. *Diferença significante, $\mathrm{P}<0,05$.

Figura 1. Média ( $\pm D P$ ) das diferentes técnicas de aterrissagem nas variáveis dependentes: (A) altura de salto, (B) tempo de contato e (C) impacto.

Tabela 1. Variação percentual e tamanho do efeito das comparações entre técnicas para cada variável dependente.

\begin{tabular}{|c|c|c|c|}
\hline Variável & Técnicas & Tamanho do efeito & $\Delta \%$ \\
\hline \multirow[t]{6}{*}{ Altura de salto } & BDJ x DJ90 & 2,39 (grande) & $39,1 \%$ \\
\hline & BDJ x DJ135 & 3,05 (grande) & $48,3 \%$ \\
\hline & $B D J \times D J 135 A$ & 3,21 (grande) & $47 \%$ \\
\hline & DJ90 x DJ135 & 0,83 (moderado) & $15,1 \%$ \\
\hline & DJ90 x DJ135A & 0,74 (pequeno) & $12,9 \%$ \\
\hline & DJ135 x DJ135A & 0,14 (trivial) & $2,5 \%$ \\
\hline \multirow[t]{6}{*}{ Tempo de contato } & BDJ x DJ90 & 4,00 (grande) & $41,3 \%$ \\
\hline & BDJ x DJ135 & 4,80 (grande) & $46,4 \%$ \\
\hline & $\mathrm{BDJ} \times \mathrm{DJ} 135 \mathrm{~A}$ & 3,74 (grande) & $48,2 \%$ \\
\hline & DJ90 x DJ135 & 1,11(moderado) & $10,3 \%$ \\
\hline & DJ90 x DJ135A & 0,9 (moderado) & $11,7 \%$ \\
\hline & DJ135 x DJ135A & 0,12 (trivial) & $1,5 \%$ \\
\hline \multirow[t]{6}{*}{ Impacto } & BDJ x DJ90 & 1,75 (grande) & $23,4 \%$ \\
\hline & BDJ x DJ135 & 0,96 (moderado) & $16 \%$ \\
\hline & $\mathrm{BDJ} \times \mathrm{DJ} 135 \mathrm{~A}$ & 0,90 (moderado) & $14 \%$ \\
\hline & DJ90 x DJ135 & 0,54 (pequeno) & $8,8 \%$ \\
\hline & DJ90 x DJ135A & 0,75 (pequeno) & $10,9 \%$ \\
\hline & DJ135 x DJ135A & 0,12 (trivial) & $2,2 \%$ \\
\hline
\end{tabular}


Concluindo que a extensão do quadril e a principal responsável pelo desempenho final dos saltos máximos. Portanto, técnicas que envolvem maior deslocamento vertical (consequentemente, maior impulso) apresentam maiores resultados na altura de salto vertical.

O desempenho do drop jump depende primariamente da distância de deslocamento do centro de massa durante a fase de absorção e propulsão de cada técnica de salto ${ }^{16}$, da velocidade de deslocamento e da otimização dos mecanismos responsáveis pelo ciclo alongamento-encurtamento (CAE) com um breve tempo de contato ${ }^{1,4}$. Como o músculo esquelético apresenta característica mecânica comprimento-dependente ${ }^{1,25,19}$, a maior amplitude articular e maior tempo de contato, observada nas técnicas DJ135 e DJ135A, podem ter dificultando a produção máxima de potência nos músculos extensores durante a execução do salto máximo. Komi ${ }^{2,4}$ e Zatsiorsky e Kraemer ${ }^{1}$ propõe que a maximização do CAE requer três condições fundamentais: ( I ) pré-ativação muscular antes da fase excêntrica, (II) fase excêntrica curta e rápida e (III) transição imediata entre as fases excêntrica e concêntrica. Juntos, tais mecanismos aperfeiçoam a resposta reflexa ao estiramento e a liberação da energia elástica acumulada pelo músculo na fase excêntrica. Ghelleher et al. ${ }^{20}$, demonstraram melhor desempenho em saltos com contra-movimento utilizando mais que $90^{\circ}$ de flexão do joelho em comparação com saltos utilizando menos de $90^{\circ}$. Adicionalmente, Salles et al. ${ }^{21}$, demonstraram maior desempenho em saltos com $90^{\circ}$ de flexão do joelho em comparação com menores flexões $\left(50\right.$ e $\left.70^{\circ}\right)$. Hahn et al. ${ }^{22}$, demonstraram que em atividades multiarticulares, o pico de torque interno produzido na articulação do joelho aproxima-se dos 50 de flexão, além de que as técnicas DJ135 e DJ135A necessitam de maior tempo para a transição entre as fases excêntrica e concêntrica e afetam negativamente o CAE pela dissipação de energia 4 .

Para o pico de impacto (normalizado pelo peso corporal) a técnica BDJ apresentou valores significantemente maiores quando comparados às técnicas DJ90 e DJ135. Como reportado na literatura, altos valores de força de reação do solo vertical são observados em saltos utilizando somente os flexores plantares ${ }^{13,21}$. Possivelmente, a manutenção dos joelhos em extensão durante a técnica BDJ foi a responsável pela maior rigidez do membro inferior durante a fase de aterrissagem. Adicionalmente, os estudos de Golhofer et al. ${ }^{23}$, e Cappa e Behm²4 reportam que os primeiros $40 \mathrm{~ms}$ de contato durante o drop jump não são controlados pelos músculos envolvidos, mas sim pela pré-ativação muscular decorrente da tentativa de aumentar a rigidez articular. Salles et al. ${ }^{21}$, e Zhang et al. ${ }^{13}$, demonstraram maior pico de impacto em saltos com contra-movimento em amplitude reduzida de flexão do joelho, sendo que o estudo de Zhang et al. ${ }^{13}$, comparou a dissipação de energia durante diferentes alturas de queda com a rigidez dos membros inferiores. Seu estudo demonstrou maior trabalho mecânico excêntrico dos extensores do joelho e quadril nos saltos mais suaves em comparação com os mais rígidos. Sendo os flexores plantares menos capazes de absorver e dissipar energia quando comparados com os extensores do joelho e quadril.

O presente estudo apresenta algumas limitações como à possível análise da atividade muscular nas diferentes técnicas de aterrissagem e a análise da altura do salto pelo cálculo da velocidade de takeoff, mesmo sendo utilizada por diversos estudos. Quanto às aplicações práticas, o entendimento das características de desempenho e mecânicas de cada técnica de aterrissagem pode auxiliar os preparadores físicos, técnicos ou fisioterapeutas na correta prescrição de acordo com a especificidade de cada modalidade, necessidade ou mesmo quanto à relação estresse $x$ desempenho. Em atividades com rápida utilização do CAE como sprints, saltos em altura e em distância, a técnica BDJ parece ser a mais indicada por apresentar ênfase nos flexores plantares e rápida transição entre as fases concêntrica e excêntrica. Em atividades com maior participação dos extensores de joelho e quadril e com maior tempo de contato com o solo, como em saltos de bloqueio e ataque no voleibol, as técnicas com maior flexão do joelho parecem ser favoráveis por apresentarem maior altura final de salto.

\section{CONCLUSÃO}

A realização de diferentes técnicas de aterrissagem podem alteram as forças de reação do solo vertical, modificando a relação estresse $x$ desempenho. Os melhores desempenhos na altura do salto vertical foram observados nas técnicas DJ90, DJ135 e DJ135A. O menor tempo de contato foi observado na técnica BDJ, seguido de DJ90, sendo que as técnicas DJ135 e DJ135A não apresentaram diferenças entre si. Quanto ao impacto, a técnica BDJ apresentou os maiores valores.

Todos os autores declararam não haver qualquer potencial conflito de interesses referente a este artigo.

CONTRIBUIÇÕES DOS AUTORES: Cada autor contribuiu individual e significativamente para o desenvolvimento do manuscrito. PHM (0000-0002-2016-936X)* contribuiu com a concepção, desenho do trabalho, análise estatística, coleta de dados, revisão e conceito intelectual do estudo. TTS (0000-0002-1208-0218)* e JJS (0000-0003-27094468)* participaram da redação e realização das coletas. EGS (0000-0002-8168-9828)*, EPS (0000-0003-2650-1949)*, RAM (0000-0002-6595-4230)*, MMSM (0000-00024785-3305)* e GBVJ (0000-0001-8136-1913)* participaram da revisão do artigo e realização das coletas. WAG (0000-0003-4223-8917)* participou da redação, revisão do manuscrito, realização das coletas do conceito intelectual do manuscrito. *ORCID (Open Researcher and Contributor ID).

\section{REFERÊNCIAS}

1. Zatsiorsky VM Kraemer WJ. Ciência e prática do treinamento de força. $2^{\text {a }}$. ed. São Paulo: Phorte Editora; 2008

2. Komi PV. Força e potência no esporte. 2. ed. São Paulo: Artmed; 2006.

3. de Villarreal ES, Kellis E, Kraemer WJ, Izquierdo M. Determining variables of plyometric training for improving vertical jump height performance: a meta-analysis. J Strength Cond Res. 2009;23(2):495-506

4. Komi PV. Stretch-shortening cycle: a powerful model to study normal and fatigued muscle. J Biomech. 2000;33(10):1197-206

5. Ebben WP, Fauth ML, Garceau LR, Petushek EJ. Kinetic quantification of plyometric exercise intensity. J Strength Cond Res. 2011;25(12):3288-98

6. Ebben WP, Simenz $C$, Jensen RL. Evaluation of plyometric intensity using electromyography. J Strength Cond Res. 2008;22(3):861-8

7. Ball NB, Stock CG, Scurr JC. Bilateral contact ground reaction forces and contact times during plyometric drop jumping. J Strength Cond Res. 2010;24(10):2762-9.

8. Peng HT. Changes in biomechanical properties during drop jumps of incremental height. J Strength Cond Res. 2011;25(9):2510-8.

9. National Strength \& Conditioning Association (NSCA), Hoffman J. Guide to Program Design (Science of Strength and Conditioning). Champaign. IL: Human Kinetics; 2012

10. Chandler TJ, Brown LE. Conditioning for strength and human performance. 2nd ed. Philadelphia: Lippincott; 2013

11. Fisher H, Stephenson ML, Graves KK, HinshawTJ, Smith DT, Zhu Q et al. Relationship between force production during isometric squats and knee flexion angles during landing. J Strength Cond Res. 2016;30(6):1670-9.

12. Devita P, Skelly WA. Effect of landing stiffness on joint kinetics and energetics in the lower extremity. Med Sci Sports Exerc. 1992;24(1):108-15.

13. Zhang SN, Bates BT, Dufek JS. Contributions of lower extremity joints to energy dissipation during landings. Med Sci Sports Exerc. 2000;32(4):812-9.
14. Rhea MR. Determining the magnitude of treatment effects in strength training research through the use of the effect size. J Strength Cond Res. 2004;18(4):918-20.

15. Rosner B. Fundamentals of biostatistics. 7th ed. Boston: Cengage Learning; 2011.

16. McBride JM, Kirby TJ, Haines TL, Skinner J. Relationship between relative net vertical impulse and jump height in jump squats performed to various squat depths and with various loads. Int J Sports Physio Perform. 2010;5(4):484-96.

17. Bobbert MF, Mackay M, Schinkelshoek D, Huijing PA, van Ingen Schenau GJ. Biomechanical analysis of drop and countermovement jumps. Eur J Appl Physiol Occup Physiol. 1986;54(6):566-73.

18. Lees A, Vanrenterghem J, De Clercq D. The maximal and submaximal vertical jump: implications for strength and conditioning. J Strength Cond Res. 2004;18(4):787-91.

19. Ide BN, Lopes CR, Sarraipa MF. Fisiologia do treinamento esportivo: força, potência, velocidade, resistência, periodização e habilidades psicológicas. São Paulo: Phorte; 2010.

20. Gheller RG, Pupo JD, Pereira LAP, Moura BMS, Santos G. A influência da profundidade de agachamento no desempenho e em parâmetros biomecânicos do salto com contramovimento. Rev Bras Cineantropom Desempenho Hum. 2014;16(6):658-68.

21. Salles AS, Baltzopoulos V, Rittweger J. Differential effects of countermovement magnitude and volitional effort on vertical jumping. Eur J Appl Physiol. 2011;111(3):441-8.

22. Hahn D, Olvermann M, Richtberg J, Seiberl W, Schwirtz A. Knee and ankle joint torque-angle relationships of multi-joint leg extension. J Biomech. 2011;44(11):2059-65.

23. Gollhofer A, Strojnik V, Rapp W, Schweizer L. Behaviour of triceps surae muscle-tendon complex in different jump conditions. Eur J Appl Physiol Occup Physiol. 1992;64(4):283-91.

24. Cappa DF, Behm DG. Neuromuscular characteristics of drop and hurdle jumps with different types of landings. J Strength Cond Res. 2013;27(11):3011-20. 